\title{
Parâmetros de degradação ruminal e digestibilidade in vitro de dietas para ruminantes utilizando diferentes níveis de coprodutos industriais
}

\author{
Mariane Moreno FERRO ${ }^{1}$, Daiane Caroline de MOURA ${ }^{2 *}$, Fabiana Gomes da COSTA², \\ Edimar Barbosa de OLIVEIRA ${ }^{1}$, Rafael Moreno FERRO ${ }^{1}$, Luciano da Silva CABRAL ${ }^{1}$ \\ 1Programa de Pós-Graduação em Ciência Animal, Universidade Federal de Mato Grosso, Cuiabá, MT, Brasil. \\ (Orcid: 0000-0001-6385-4618; 0000-0002-5003-5581; 0000-0003-2954-5162; 0000-0001-6385-4618) \\ 2Universidade do Estado de Mato Grosso, Pontes e Lacerda, MT, Brasil. \\ 3 Programa de Pós-Graduação em Zootecnia, Universidade Federal de Goiás, Goiânia, GO, Brasil. (Orcid: 0000-0002-9539-879X) \\ *E-mail: daiane_caroline@zootecnista.com.br (Orcid: 0000-0002-8276-2089)
}

Recebido em 28/04/2020; Aceito em 03/08/2020; Publicado em 26/08/2020.

\begin{abstract}
RESUMO: Objetivou-se investigar diferentes níveis de inclusão de três coprodutos (farelo de gérmen de milho desengordurado, grão de destilaria de milho e farelo de girassol) sob os parâmetros de degradação ruminal e digestibilidade in vitro. As dietas experimentais foram constituídas pela inclusão de níveis de 0, 100, 200 e 300 $\left(\mathrm{g} \mathrm{kg}^{-1}\right)$ dos coprodutos. A inclusão farelo de gérmen de milho desengordurado não alterou $(P>0,05)$ a produção de gases da fração CNF, a taxa de degradação da fração CNF e a taxa de degradação da fração fibrosa, enquanto, a produção de gases da fração fibrosa apresentou redução linear $(P<0,05)$. A inclusão do grão de destilaria de milho não alterou $(P>0,05)$ a digestibilidade da MS e a taxa de degradação da fração rápida digestão, entretanto a produção de gases da fração de rápida digestão $(V G C N F)$ apresentou redução linear $(P<0,05)$. A inclusão do farelo de girassol não alterou $(P>0,05)$ a produção de gases, a taxa de degradação da fração CNF e digestibilidade da MS. Assim o grão seco da destilaria do milho e o farelo de girassol podem ser incluídos até o nível de $300 \mathrm{~g} \mathrm{~kg}^{-1}$ nas dietas, e o farelo de gérmen de milho desengordurado incluído em níveis de até $100 \mathrm{~g}$ $\mathrm{kg}^{-1}$.

Palavras-chave: degradação ruminal; farelo de girassol; farelo de gérmen de milho; grão seco da destilaria do milho.
\end{abstract}

\section{Parameters of ruminal degradation and in vitro digestibility of diets for ruminants using different levels of industrial co-products}

\begin{abstract}
The objective was to investigate different levels of inclusion of three co-products (defatted corn germ meal, corn distillery grain and sunflower meal) under the parameters of ruminal degradation and in vitro digestibility. The experimental diets were constituted by the inclusion of levels of 0, 100, 200 and $300\left(\mathrm{~g} \mathrm{~kg}^{-1}\right)$ of the co-products. The inclusion of defatted corn germ meal no effect $(\mathrm{P}>0.05)$ the production of NFC fraction gases, the NFC fraction degradation rate and the fibrous fraction degradation rate, while the production of fibrous fraction gases presented a linear reduction $(\mathrm{P}<0.05)$. The inclusion of the corn distillery grain no affect $(\mathrm{P}>0.05)$ the digestibility of $\mathrm{DM}$ and the rate of degradation of the fast digest fraction, however the production of gases of the fast digest fraction showed a linear reduction $(\mathrm{P}<0.05)$. The inclusion of sunflower meal no effect $(\mathrm{P}>0.05)$ the gas production, the NFC fraction degradation rate and DM digestibility. Thus, the dry grain of the corn distillery and sunflower meal can be included up to the level of $300 \mathrm{~g} \mathrm{~kg}^{-1} \mathrm{in}_{\text {the diets, and }}$ the defatted corn germ meal included in levels of up to $100 \mathrm{~g} \mathrm{~kg}^{-1}$.

Key words: ruminal degradation; sunflower meal; corn germ meal; dry grain from corn distillery.
\end{abstract}

\section{INTRODUÇÃO}

A utilização de coprodutos agroindustriais na alimentação de ruminantes apresenta como vantagens o aumento na oferta de ingredientes tradicionalmente utilizados em rações de não ruminantes e alimentação humana, como milho e soja, uma vez que a inclusão desses coprodutos pode reduzir a dependência de utilização total ou parcial desses ingredientes. Outro ponto é a possibilidade de negociação de preços com consequente otimização dos custos, uma vez que os coprodutos não se apresentam como commodities e colaboram com a redução da poluição ambiental, já que, a forma de descarte desses alimentos pela indústria ainda não está estabelecida (PAIXÃO, 2008).
A avaliação do consumo e digestibilidade dos nutrientes e seu comportamento nos diferentes compartimentos do trato digestivo são de extrema importância para obtenção de um valor nutritivo dos alimentos (ARMENTO; PEREIRA, 1997). Um dos principais objetivos na nutrição de ruminantes é maximizar a produção de proteína microbiana através da sincronização da degradação de energia e proteína no rúmen.

$\mathrm{O}$ valor nutritivo de um alimento depende de diversos fatores, entre eles a sua composição química e o aproveitamento de seus nutrientes pelos animais, uma vez que, em ruminantes esse aproveitamento é resultante da simbiose entre o animal e a microbiota ruminal (SANTO et al., 2017). Assim, o valor nutritivo de um alimento não pode ser considerado como fator isolado, mas como um complexo 
formado por diversos fatores que interagem, e podem interferir na ingestão e utilização dos alimentos consumidos pelos ruminantes.

Desta forma, o conhecimento da dinâmica de degradação ruminal de diversos alimentos é fundamental para adequação das dietas quanto as informações relativas às proporções das frações dos alimentos, bem como sua taxa de degradação e perdas decorrentes da fermentação ruminal (GOES et al., 2010). As técnicas de incubação in vitro, com a utilização de inóculos ruminais têm sido descritas como alternativas às técnicas in vivo e in situ para avaliar o padrão de digestão dos alimentos (TILLEY; TERRY, 1963), principalmente devido à sua facilidade de uso e ao seu baixo custo e com obtenção de estimativas acuradas dos parâmetros de digestão (HUHTANEN et al., 2008).

A utilização dos coprodutos agroindustrial está bem consolidada na nutrição de ruminantes. Entretanto, muitos coprodutos agroindustriais não apresentam informações suficientes quanto a sua composição química e níveis adequados de inclusão, levando a necessidade de estudar a sua viabilidade de utilização e quantificar as respostas nutricionais e fisiológicas em ruminantes (LOUSADA JUNIOR et al., 2005).

Neste contexto, hipotetizamos que estes coprodutos ao serem incluídos em dietas para ruminantes até o nível de 300 $\mathrm{g} \mathrm{kg}^{-1}$ não irão afetar os parâmetros de degradação e a digestibilidade ruminal in vitro da matéria seca. Assim, objetivou-se investigar diferentes níveis de inclusão desses coprodutos sob os parâmetros de degradação ruminal e digestibilidade in vitro.

\section{MATERIAL E MÉTODOS}

\subsection{Local e animais}

As incubações foram realizadas nas dependências do laboratório de Nutrição Animal da Universidade Federal de Mato Grosso - Campus Cuiabá-MT. Foram utilizados dois ovinos adultos, canulados no rúmen para coleta do líquido ruminal, mantidos no setor de Experimentação de Ruminantes na cidade de Santo Antônio do Leverger, pertencente à Universidade Federal de Mato Grosso.

\subsection{Dietas experimentais e análises químico- bromatológicas}

As dietas experimentais e a ofertadas para os animais foram compostas por $30 \%$ de volumoso (silagem de milho) e $70 \%$ de concentrado, na matéria seca, sendo as mesmas isoprotéicas com 16\% de PB (NRC, 2007). Os tratamentos foram constituídos pela inclusão de 0, 100, 200 e $300 \mathrm{~g} \mathrm{~kg}^{-1}$ dos três diferentes coprodutos nas dietas experimentais. A participação dos ingredientes nas dietas está demonstrada na Tabela 1.

Todas as amostras foram analisadas de acordo com procedimentos de análise padrão do Instituto Nacional Brasileiro de Ciência e Tecnologia na Ciência Animal (INCTCA) (DETMANN et al., 2012).

A amostra da silagem de milho foi pré-seca em estufa de ventilação forçada sob $55^{\circ} \mathrm{C}$ durante 72 horas para determinação da amostra seca ao ar (INCT-CA G-001/1). Posteriormente, todos os alimentos foram moídos em peneira de $1 \mathrm{~mm}$. Em seguida todos os alimentos foram submetidos a estufa à $105^{\circ} \mathrm{C}$ para determinação da matéria seca (MS definitiva) (INCT-CA G-003/1), proteína bruta PB (INCT-CA N-001/1), extrato etéreo (EE) (INCT-CA G-
004/1), fibra detergente neutro (FDN) (INCT-CA F-002/1), sendo o resíduo da FDN corrigido para cinzas e compostos nitrogenados (FDNcp) determinado conforme Licitra et al. (1996). Os CNF foram calculados conforme proposto por Hall (2003). A composição químico-bromatológica dos ingredientes está demonstrada na Tabela 2.

Tabela 1. Composição das dietas experimentais.

Table 1. Composition of experimental diets.

\begin{tabular}{|c|c|c|c|c|}
\hline \multirow[t]{2}{*}{ Ingrediente } & \multicolumn{4}{|c|}{ Níveis de inclusão $\left(\mathrm{g} \mathrm{kg}^{-1}\right)$} \\
\hline & 0 & 100 & 200 & 300 \\
\hline \multicolumn{5}{|c|}{ Farelo de Gérmen de milho desengordurado } \\
\hline Silagem de milho & 300 & 300 & 300 & 300 \\
\hline Milho & 500 & 400 & 310 & 220 \\
\hline $\begin{array}{l}\text { Farelo de gérmen de } \\
\text { milho desengordurado }\end{array}$ & 000 & 110 & 210 & 320 \\
\hline Farelo de soja & 200 & 190 & 180 & 160 \\
\hline \multicolumn{5}{|c|}{ Grão seco da destilaria do milho } \\
\hline Silagem de milho & 300 & 300 & 300 & 300 \\
\hline Milho & 500 & 460 & 420 & 380 \\
\hline $\begin{array}{l}\text { Grão seco destilaria do } \\
\text { milho }\end{array}$ & 000 & 110 & 210 & 320 \\
\hline Farelo de soja & 200 & 130 & 70 & 000 \\
\hline \multicolumn{5}{|c|}{ Farelo de girassol } \\
\hline Silagem de milho & 300 & 300 & 300 & 300 \\
\hline Milho & 500 & 460 & 420 & 380 \\
\hline Farelo de girrassol & 000 & 110 & 210 & 320 \\
\hline Farelo de soja & 200 & 130 & 70 & 000 \\
\hline
\end{tabular}

Tabela 2. Composição químico-bromatológica dos ingredientes que constituíram as dietas experimentais.

Table 2. Chemical-chemical composition of the ingredients that constituted the experimental diets.

\begin{tabular}{|c|c|c|c|c|c|c|}
\hline \multirow{2}{*}{ Componentes } & \multicolumn{6}{|c|}{$\operatorname{Alimentos}^{1}\left(\mathrm{~g} \mathrm{~kg}^{-1} \mathrm{MS}\right)$} \\
\hline & SM & MI & FS & FGMD & DDGM & FG \\
\hline Matéria seca & 300 & 870 & 910 & 900 & 910 & 910 \\
\hline Proteína bruta & 90 & 80 & 540 & 110 & 360 & 390 \\
\hline Extrato etéreo & 20 & 50 & 20 & 50 & 70 & 20 \\
\hline $\begin{array}{l}\text { Fibra detergente } \\
\text { neutro }\end{array}$ & 550 & 80 & 110 & 250 & 130 & 450 \\
\hline $\begin{array}{l}\text { Carboidratos não- } \\
\text { fibrosos }\end{array}$ & 310 & 630 & 140 & 450 & 90 & 200 \\
\hline
\end{tabular}

${ }^{1}$ SM: silagem de milho; FS: farelo de soja; MI: milho moído; FGMD: farelo de gérmen de milho desengordurado; DDGM: grão seco da destilaria do milho e FG: farelo de girassol.

\subsection{Ensaios in vitro}

Em cada incubação foram utilizadas duas repetições por tratamento, sendo efetuadas três incubações sucessivas, totalizando seis frascos por tratamento. Cada incubação foi utilizada como bloco, e das repetições dos tratamentos dentro de cada bloco foram efetuadas médias.

Para desenvolvimento das incubações, os alimentos foram moídos a $1 \mathrm{~mm}$ conforme Pell; Schofield, (1993) em seguida foram pesados $500 \mathrm{mg}$ de amostra seca ao ar (ASA) e as amostras alocadas em frascos de vidro cor âmbar com capacidade de $120 \mathrm{~mL}$.

O meio utilizado foi composto seguindo recomendação de McDougall (1949), preparado para manter o pH entre 6,9 a 7,0, com aspersão constante de $\mathrm{CO}_{2}$. O inóculo ruminal foi coletado individualmente de cada ovino, duas horas após a alimentação matinal, e filtrados com auxílio de sacos de tecido, e acondicionando em garrafa térmica pré-aquecida com fechamento hermético e transportado imediatamente para o laboratório sendo toda a sua manipulação realizada sob aspersão de $\mathrm{CO}_{2}$ (livre de oxigênio). 
Posteriormente o material foi filtrado em quatro camadas de gaze, e foi efetuado uma mistura das amostras de líquido, formando uma amostra composta, e mantido a $39^{\circ} \mathrm{C}$ por 10 minutos em repouso em frasco transparente com infusão contínua de $\mathrm{CO}_{2}$.

Aos frascos foram adicionados $40 \mathrm{~mL}$ de solução meio de McDougall e imediatamente receberam tampa de borracha e lacre de alumínio. Após meia hora, foram adicionados 10 $\mathrm{mL}$ de inóculo ruminal sob aspersão de $\mathrm{CO}_{2}$ utilizando seringa e agulha hipodérmica ( $40 \times 12 \mathrm{~mm}) ;\left(18 \mathrm{G} \times 1 \frac{1}{1 / 2}\right.$ ") e mantidos à temperatura de $39^{\circ} \mathrm{C}$ em banho-maria com agitação orbital constante (MALAFAIA, 1997).

\subsection{Produção total de gases}

A produção total de gases foi mensurada utilizando sistema semi-automático (THEODOROU et al., 1994), por meio de transdutor de pressão (psi - pressão por polegada quadrada) (Modelo DPI 705 Series Digital; Marca GE, Leicester, Inglaterra) para aferir a pressão absoluta em mega pascal (MPa) nos seguintes tempos: 1, 2, 3, 4, 5, 6, 8, 10, 12, 24, 36, 48, 60, 72 e 96 horas. Para descontar o volume de gases oriundo do líquido de rúmen e da solução tampão, dois frascos foram incubados sem amostra (branco). Dessa forma, para cada tempo de leitura, o volume de gases dos frascos com amostra foi subtraído do volume dos frascos sem amostra.

Com o somatório do volume de gases para cada tempo de leitura, foram estabelecidas as curvas de produção cumulativa dos gases. A conversão de psi para $\mathrm{mL}$ foi feita a partir da equação de regressão linear $(\mathrm{Y}=\mathrm{a} \pm \mathrm{bx})$ em que o coeficiente "b" da equação possibilitou a correção e transformação de pressão (psi) em volume de gases $(\mathrm{mL})$ corrigido para a pressão barométrica do dia. Para isso, injetou-se volume conhecido de gases em frascos mantidos sob as mesmas condições das amostras incubadas. As medidas tomadas foram utilizadas para a obtenção da equação de regressão entre pressão e volume de gases.

A produção cumulativa dos gases foi analisada empregando-se o modelo logístico bicompartimental descrito por Schofield et al. (1994) onde: $\mathrm{V}(\mathrm{t})=\mathrm{Vf}_{1} /(1+$ $\left.\exp \left(2-4^{*} \mathrm{c}_{1} *(\mathrm{~T}-\mathrm{L})\right)\right)+\mathrm{Vf}_{2} /\left(1+\exp \left(2-4^{*} \mathrm{c}_{2} *(\mathrm{~T}-\mathrm{L})\right)\right)$, no qual $\mathrm{V}(\mathrm{t})$ é o volume de gases acumulado no tempo $\mathrm{t}$; $\mathrm{Vf}_{1}$, o volume de gases oriundo da fração de rápida degradação (fração $\mathrm{CNF}) ; \mathrm{c}_{1}\left(\mathrm{~h}^{-1}\right)$, a taxa de degradação da fração de rápida degradação; $\mathrm{T}$, o tempo (h); $\mathrm{L}$, a latência; $\mathrm{Vf}_{2}$, o volume de gases da fração de lenta degradação (fração carboidratos fibrosos); e $c_{2}\left(h^{-1}\right)$, a taxa de degradação da fração de lenta degradação.

\subsection{Estimativa de digestibilidade in vitro}

Para estimativa da digestibilidade da MS das dietas experimentais e dos alimentos, ao término de cada incubação o resíduo da digestão in vitro foi filtrado utilizando-se cadinhos filtrantes e em seguida colocados em estufa à $105^{\circ} \mathrm{C}$ para determinação da MS (método $\mathrm{N}^{\circ}$ 934.01) (AOAC, 1990). A digestibilidade da MS foi calculada dividindo-se o resíduo da incubação pelo total de amostra incubada, expresso em $\%$.

\subsection{Análise estatística}

Os efeitos de tratamentos foram decompostos em três contrastes ortogonais: efeito linear, efeito quadrático e efeito cúbico dos níveis de inclusão dos coprodutos $(0,100,200$ e $\left.300 \mathrm{~g} \mathrm{Kg} \mathrm{Kg}^{-1}\right)$. Adotou-se nível crítico de $P<0,05$ de probabilidade para o erro tipo I, utilizando o Proc Mixed do SAS versão 9.2

\section{RESULTADOS}

\subsection{Parâmetros de degradação e digestibilidade dos ingredientes}

Dentre os coprodutos avaliados, o farelo de gérmen de milho desengordurado apresentou uma produção total de gases superior em todas as horas de incubação. Nas primeiras 24 horas de incubação a produção de gases do farelo de girassol manteve-se acima da produção de gases do grão seco da destilaria do milho, porém, a partir das 24 horas de incubação a produção de gases entre os dois coprodutos inverteu.

Dentre os alimentos que constituíram as dietas experimentais, a produção de gases da fração de rápida degradação (VGCNF) foi numericamente maior para a silagem de milho $(39,14 \mathrm{~mL})$, seguida do farelo de soja $(38,70$ $\mathrm{mL})$ e do farelo de gérmen de milho desengordurado $(38,57$ $\mathrm{mL})$, e menor para farelo de girassol $(21,66 \mathrm{~mL})$, milho $(18,55$ $\mathrm{mL})$ e o grão seco da destilaria do milho $(8,68 \mathrm{~mL})$. Uma maior taxa de degradação desta mesma fração (KdCNF) foi observada para o milho $\left(31,38 \%\right.$ hora $\left.^{-1}\right)$, seguido do farelo de soja $\left(16,71 \%\right.$ hora $\left.^{-1}\right)$, farelo de gérmen de milho desengordurado $\left(15,88 \%\right.$ hora $\left.^{-1}\right)$ e farelo de girassol $(15,07 \%$ hora $\left.^{-1}\right)$, com menor taxa para a silagem de milho $(9,43 \%$ hora 1) e grão seco da destilaria do milho $\left(6,28 \%\right.$ hora $\left.^{-1}\right)$ (Tabela 3).

Para a fração de lenta degradação (VGCHOF) uma maior produção de gases foi observada para o milho grão $(129,20$ $\mathrm{mL})$, seguido do grão seco da destilaria do milho $(98,57 \mathrm{~mL})$, silagem de milho (77,50 mL) e farelo de soja $(75,40 \mathrm{~mL})$, com menor produção observada para o farelo de gérmen de milho desengordurado $(56,24 \mathrm{~mL})$ e o farelo de girassol $(27,99 \mathrm{~mL})$. Para esta mesma fração a maior taxa de degradação (KdCHOF) foi observada para o milho grão $\left(3,55 \%\right.$ hora $\left.^{-1}\right)$, seguido do farelo de gérmen de milho desengordurado $\left(2,77 \%\right.$ hora $\left.^{-1}\right)$, grão seco de destilaria do milho $(1,80 \%$ hora $\left.{ }^{1}\right)$, farelo de soja $\left(1,69 \%\right.$ hora $\left.^{-1}\right)$, farelo de girassol $(1,60 \%$ hora $\left.^{-1}\right)$ e silagem de milho $\left(1,34 \%\right.$ hora $\left.^{-1}\right)$ (Tabela 3$)$.

O volume total de gases foi maior para milho grão (147, $75 \mathrm{~mL})$, seguido da silagem de milho $(116,64 \mathrm{~mL})$ e farelo de soja $(114,10 \mathrm{~mL})$, com menor produção para grão seco da destilaria do milho $(107,25 \mathrm{~mL})$, farelo de gérmen de milho desengordurado $(94,81 \mathrm{~mL})$ e farelo de girassol $(49,65 \mathrm{~mL})$. Uma maior latência foi observada para o grão seco da destilaria do milho (13,48 horas), com menor latência para farelo de girassol (1,84 horas).

A digestibilidade ruminal in vitro da matéria seca foi maior para milho grão com 95,33\%, e dentre os coprodutos avaliados, o grão seco da destilaria do milho foi o que apresentou maior digestibilidade ruminal com 63,95\% (Tabela 3).

\subsection{Parâmetros de degradação e digestibilidade dos ingredientes \\ 3.2.1. Farelo de gérmen de milho desengordurado}

A Figura 1 mostra que os níveis de inclusão de farelo de gérmen de milho desengordurado nas primeiras 12 horas de fermentação foram bastante similares. Entretanto, a inclusão de 0 e $100 \mathrm{~g} \mathrm{~kg}^{-1}$ de farelo de gérmen de milho desengordurado aumentou consideravelmente a produção de gases após as 12 horas de fermentação. 
A produção de gases (VGCNF) e a taxa de degradação da fração de rápida digestão $(\mathrm{KdCNF})$ não apresentaram efeito significativo $(\mathrm{P}>0,05)$ com a inclusão do farelo de gérmen de milho desengordurado nas dietas. A produção de gases da fração de lenta digestão (VGCHOF) apresentou redução linear $(\mathrm{P}<0,05)$, enquanto, a taxa de degradação desta mesma fração (KdCHOF) não apresentou diferença significativa ( $\mathrm{P}>0,05)$ com os níveis de inclusão do farelo de gérmen de milho desengordurado. A latência e a produção total de gases
(VGtotal) reduziu linearmente $(\mathrm{P}<0,05)$ com a inclusão do farelo de gérmen de milho desengordurado (Tabela 4).

Houve redução linear $(\mathrm{P}<0,05)$ na digestibilidade ruminal in vitro com a inclusão dos níveis deste coproduto, constituíram devido a sua reduzida taxa de degradação comparativamente ao milho e farelo de soja que foram os alimentos substituídos à medida que foi incluído este coproduto.

Tabela 3. Parâmetros de degradação e digestibilidade ruminal in vitro da matéria seca dos ingredientes que constituíram as dietas experimentais.

Table 3. Degradation parameters and in vitro ruminal digestibility of the dry matter of the ingredients that constituted the experimental diets.

\begin{tabular}{lcccccc}
\hline \multirow{2}{*}{ Parâmetros ${ }^{1}$} & \multicolumn{9}{c}{ Alimentos dietéticos ${ }^{2}$} \\
\cline { 2 - 7 } & SM & MI & FS & FGMD & DDGM & FG \\
\hline VGCNF & 39,14 & 18,55 & 38,70 & 38,57 & 8,68 & 21,66 \\
KdCNF & 0,0943 & 0,3138 & 0,1671 & 0,1588 & 0,0628 & 0,1507 \\
VGCHOF & 77,50 & 129,20 & 75,40 & 56,24 & 98,57 & 27,99 \\
KdCHOF & 0,0134 & 0,0355 & 0,0169 & 0,0277 & 0,0180 & 0,0160 \\
Latência & 4,34 & 3,90 & 3,66 & 3,60 & 13,48 & 1,84 \\
VGtotal & 116,64 & 147,75 & 114,10 & 94,81 & 107,25 & 49,65 \\
DIVMS & 67,42 & 95,33 & 90,66 & 60,95 & 63,95 & 59,21 \\
\hline
\end{tabular}

${ }^{1}$ VGCNF (ml $\left.500 \mathrm{mg}^{-1} \mathrm{MS}\right)$ : volume final de gás referente à fração de rápida digestão (CNF); KdCNF (\%h-1): taxa de degradação referente à fração de rápida digestão $(\mathrm{CNF})$; VGCHOF ( $\left.\mathrm{ml} 500 \mathrm{mg}^{-1} \mathrm{MS}\right)$ : volume final de gás referente à fração de lenta digestão $(\mathrm{CHOF}) ; \mathrm{KdCHOF}\left(\% \mathrm{~h}^{-1}\right)$ : taxa de degradação referente à fração de lenta degradação $(\mathrm{CHOF})$; Latência: horas; VGtotal: volume total de gases após 96 horas de incubação; DIVMS: digestibilidade in vitro de matéria seca após 96 horas de incubação; ${ }^{2}$ SM: silagem de milho; MI: Milho grão; FS: Farelo de soja; FGMD: Farelo de gérmen de milho desengordurado; DDGM: Grão seco da destilaria do milho; FG: Farelo de girassol

Tabela 4. Parâmetros de degradação e digestibilidade ruminal in vitro da matéria seca das dietas contendo diferentes níveis de inclusão do farelo de gérmen de milho desengordurado.

Table 4. Degradation parameters and in vitro ruminal digestibility of dry matter from diets containing different levels of defatted corn germ bran inclusion.

\begin{tabular}{|c|c|c|c|c|c|c|c|c|}
\hline \multirow[t]{2}{*}{ Parâmetros $^{1}$} & \multicolumn{4}{|c|}{ Níveis de inclusão $\left(\mathrm{g} \mathrm{kg}^{-1}\right)$} & \multirow[t]{2}{*}{ EPM } & \multicolumn{3}{|c|}{ Valor-P } \\
\hline & 0 & 100 & 200 & 300 & & Linear & Quadrático & Cúbico \\
\hline VGCNF & 45,56 & 55,52 & 50,65 & 52,72 & 8,68 & 0,3221 & 0,2943 & 0,2023 \\
\hline $\mathrm{KdCNF}$ & 0,1051 & 0,0918 & 0,0932 & 0,0920 & 0,007 & 0,1736 & 0,3159 & 0,5139 \\
\hline VGCHOF & 86,00 & 78,83 & 68,39 & 60,35 & 9,71 & 0,0019 & 0,9253 & 0,7851 \\
\hline $\mathrm{KdCHOF}$ & 0,0205 & 0,0176 & 0,0193 & 0,0179 & 0,001 & 0,4732 & 0,6866 & 0,3757 \\
\hline Latência & 5,87 & 5,65 & 5,15 & 3,73 & 0,87 & $<0,0001$ & 0,6920 & 0,0630 \\
\hline VGtotal & 131,57 & 134,37 & 119,05 & 113,08 & 11,79 & 0,0003 & 0,1513 & 0,0555 \\
\hline DIVMS & 89,82 & 84,56 & 76,02 & 75,34 & 5,13 & 0,0144 & 0,5273 & 0,4930 \\
\hline
\end{tabular}

${ }^{1}$ VGCNF (ml $\left.500 \mathrm{mg}^{-1} \mathrm{MS}\right)$ : volume final de gás referente à fração de rápida digestão $(\mathrm{CNF}) ; \mathrm{KdCNF}\left(\% \mathrm{~h}^{-1}\right)$ : taxa de degradação referente à fração de rápida digestão (CNF); VGCHOF ( $\left.\mathrm{ml} 500 \mathrm{mg}^{-1} \mathrm{MS}\right)$ : volume final de gás referente à fração de lenta digestão (CHOF); $\mathrm{KdCHOF}\left(\% \mathrm{~h}^{-1}\right)$ : taxa de degradação referente à fração de lenta degradação (CHOF); Latência: horas; VGtotal: volume total de gases após 96 horas de incubação; DIVMS: digestibilidade in vitro de matéria seca após 96 horas de incubação.

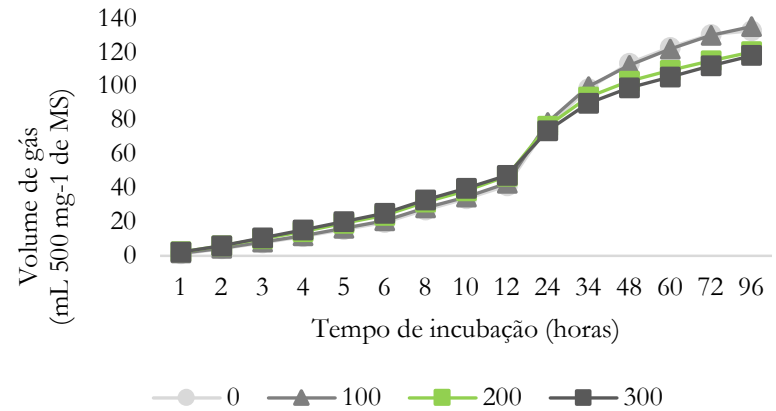

Figura 1. Produção cumulativa de gases das dietas experimentais que tiveram inclusão do farelo de gérmen de milho desengordurado. Figure 1. Cumulative gas production from experimental diets that included defatted corn germ meal

\subsubsection{Grão seco da destilaria do milho}

O volume de gases nas primeiras horas de incubação foi bastante similar entre os diferentes níveis de inclusão do grão seco de destilaria do milho (Figura 2).
Podemos observar que os níveis 0 e $100 \mathrm{~g} \mathrm{~kg}^{-1}$ de inclusão tiveram perfil de fermentação bastante similares e com produção superior aos demais níveis.

A produção de gases da fração de rápida digestão (VGCNF) apresentou redução linear $(\mathrm{P}<0,05)$ com a inclusão dos níveis de grão de destilaria do milho, entretanto, essa menor produção de gases não levou a alteração da taxa de degradação desta mesma fração (KdCNF), sem efeito significativo $(\mathrm{P}>0,05)$. A produção de gases da fração de lenta (VGCHOF) digestão não apresentou efeito significativo $(\mathrm{P}>0,05)$, porém, a taxa de degradação desta fração (KdCHOF) apresentou redução linear $(\mathrm{P}<0,05)$ com $\mathrm{O}$ aumento da inclusão deste coproduto (Tabela 5). A latência, a produção de gases total (VGtotal) e a digestibilidade ruminal in vitro da MS não apresentaram efeito significativo $(\mathrm{P}>0,05)$ com a inclusão do grão seco da destilaria do milho nas dietas (Tabela 5). 


\subsubsection{Farelo de girassol}

O volume de gases nas primeiras 12 horas de fermentação foi bastante similar entre os diferentes níveis de inclusão de farelo de girassol (Figura 3). Entretanto, observa-se que após 24 horas de fermentação a produção de gases aumentou para o tratamento de $0 \%$ de inclusão do farelo de girassol.

A produção de gases da fração de rápida digestão (VGCNF) não apresentou efeito significativo $(\mathrm{P}>0,05)$ à medida que aumentou os níveis de inclusão do farelo de girassol, seguindo o mesmo comportamento para a taxa de degradação desta mesma fração (KdCNF) (P>0,05).

A produção de gases da fração de lenta digestão (VGCHOF) apresentou comportamento quadrático $(\mathrm{P}<0,05)$, porém, a taxa de degradação $(\mathrm{KdCHOF})$ desta mesma fração não foi alterada $(\mathrm{P}>0,05)$. A latência, o volume total de gases (VGtotal) e digestibilidade ruminal in vitro da MS não apresentaram efeito significativo $(\mathrm{P}>0,05) \mathrm{com}$ a inclusão do farelo de girassol (Tabela 6).

Tabela 5. Parâmetros de degradação e digestibilidade ruminal in vitro da matéria seca das dietas contendo diferentes níveis de inclusão do grão seco da destilaria do milho.

Table 5. Degradation parameters and in vitro ruminal digestibility of dry matter from diets containing different levels of inclusion of dry grain from corn distillery.

\begin{tabular}{|c|c|c|c|c|c|c|c|c|}
\hline \multirow[t]{2}{*}{ Parâmetros ${ }^{1}$} & \multicolumn{4}{|c|}{ Níveis de inclusão $\left(\mathrm{g} \mathrm{kg}^{-1}\right)$} & \multirow[t]{2}{*}{ EPM } & \multicolumn{3}{|c|}{ Valor-P } \\
\hline & 0 & 100 & 200 & 300 & & Linear & Quadrático & Cúbico \\
\hline VGCNF & 52,66 & 53,39 & 46,33 & 38,67 & 11,71 & 0,0419 & 0,3619 & 0,7189 \\
\hline $\mathrm{KdCNF}$ & 0,0922 & 0,0762 & 0,0860 & 0,0905 & 0,018 & 0,8858 & 0,2084 & 0,3729 \\
\hline VGCHOF & 72,86 & 76,77 & 83,96 & 83,57 & 14,13 & 0,1403 & 0,6925 & 0,6559 \\
\hline $\mathrm{KdCHOF}$ & 0,0196 & 0,0185 & 0,0184 & 0,0172 & 0,002 & 0,0421 & 0,9208 & 0,4860 \\
\hline Latência & 4,65 & 4,74 & 6,28 & 7,75 & 2,04 & 0,0531 & 0,5227 & 0,7473 \\
\hline VGtotal & 125,53 & 130,17 & 130,30 & 122,25 & 23,81 & 0,4699 & 0,0648 & 0,7807 \\
\hline DIVMS & 74,31 & 68,32 & 68,33 & 63,09 & 8,36 & 0,3101 & 0,9557 & 0,7110 \\
\hline
\end{tabular}

${ }^{1}$ VGCNF (ml $\left.500 \mathrm{mg}^{-1} \mathrm{MS}\right)$ : volume final de gás referente à fração de rápida digestão $(\mathrm{CNF}) ; \mathrm{KdCNF}\left(\% \mathrm{~h}^{-1}\right)$ : taxa de degradação referente à fração de rápida digestão $(\mathrm{CNF})$; VGCHOF ( $\left.\mathrm{ml} 500 \mathrm{mg}^{-1} \mathrm{MS}\right)$ : volume final de gás referente à fração de lenta digestão $(\mathrm{CHOF}) ; \mathrm{KdCHOF}\left(\% \mathrm{~h}^{-1}\right)$ : taxa de degradação referente à fração de lenta degradação $(\mathrm{CHOF})$; Latência: horas; VGtotal: volume total de gases após 96 horas de incubação; DIVMS: digestibilidade in vitro de matéria seca após 96 horas de incubação

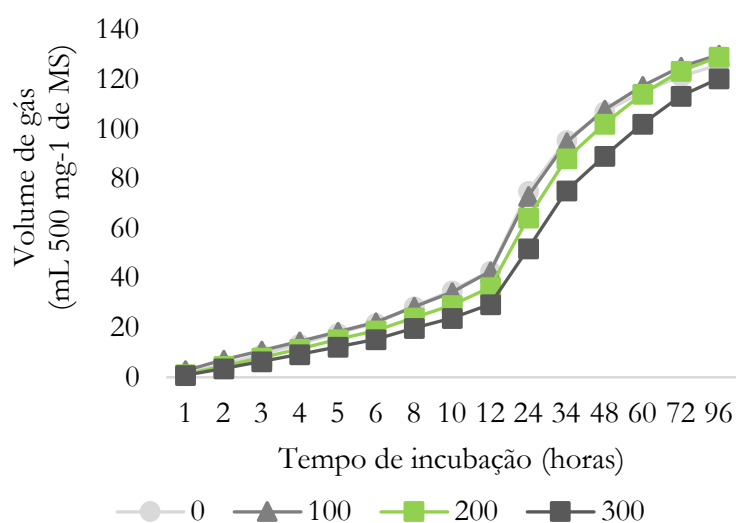

Figura 2. Produção cumulativa de gases das dietas experimentais que tiveram inclusão do grão seco de destilaria do milho.

Figure 2. Cumulative production of gases from experimental diets that included dry grain from corn distillery.

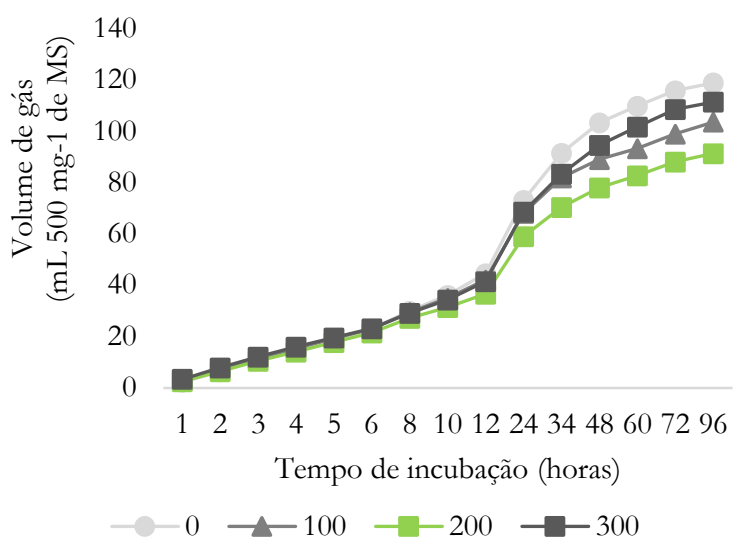

Figura 3. Produção cumulativa de gases das dietas experimentais que tiveram inclusão do farelo de girassol.

Figure 3. Cumulative gas production from experimental diets that included sunflower meal.

Tabela 6. Parâmetros de degradação e digestibilidade ruminal in vitro da matéria seca das dietas contendo diferentes níveis de inclusão do farelo de girassol.

Table 6. Degradation parameters and in vitro ruminal digestibility of dry matter from diets containing different levels of sunflower meal inclusion.

\begin{tabular}{|c|c|c|c|c|c|c|c|c|}
\hline \multirow{2}{*}{ Parâmetros ${ }^{1}$} & \multicolumn{4}{|c|}{ Níveis de inclusão $\left(\mathrm{g} \mathrm{kg}^{-1}\right)$} & \multirow[t]{2}{*}{ EPM } & \multicolumn{3}{|c|}{ Valor-P } \\
\hline & 0 & 100 & 200 & 300 & & Linear & Quadrático & Cúbico \\
\hline VGCNF & 46,65 & 49,19 & 40,72 & 45,92 & 14,36 & 0,7507 & 0,8588 & 0,4704 \\
\hline $\mathrm{KdCNF}$ & 0,0962 & 0,0896 & 0,1233 & 0,0784 & 0,028 & 0,8440 & 0,4127 & 0,2674 \\
\hline VGCHOF & 72,22 & 55,17 & 50,78 & 65,56 & 14,42 & 0,1862 & 0,0048 & 0,7044 \\
\hline $\mathrm{KdCHOF}$ & 0,0206 & 0,0219 & 0,0215 & 0,0170 & 0,005 & 0,5285 & 0,4792 & 0,8902 \\
\hline Latência & 4,27 & 3,25 & 3,17 & 3,44 & 0,66 & 0,0874 & 0,0603 & 0,6576 \\
\hline VGtotal & 118,97 & 104,46 & 91,63 & 111,57 & 20,81 & 0,3216 & 0,0552 & 0,3754 \\
\hline DIVMS & 70,56 & 76,72 & 78,71 & 73,75 & 7,57 & 0,6241 & 0,3129 & 0,9083 \\
\hline
\end{tabular}

${ }^{1}$ VGCNF (ml $\left.500 \mathrm{mg}^{-1} \mathrm{MS}\right)$ : volume final de gás referente à fração de rápida digestão $(\mathrm{CNF}) ; \mathrm{KdCNF}\left(\% \mathrm{~h}^{-1}\right)$ : taxa de degradação referente à fração de rápida

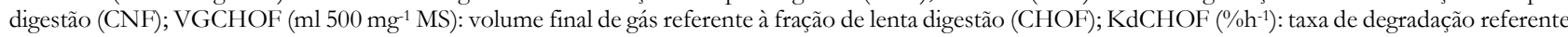
à fração de lenta degradação (CHOF); Latência: horas; VGtotal: volume total de gases após 96 horas de incubação; DIVMS: digestibilidade in vitro de matéria seca após 96 horas de incubação

\section{DISCUSSÃO}

O comportamento para os parâmetros de degradação e digestibilidade dos ingredientes pode ser explicado pela característica fibrosa dos mesmos, nos quais os alimentos com maior teor de amido e carboidratos mais rapidamente fermentáveis apresentaram maior produção de gases e maior digestibilidade isso ocorre devido melhor ataque dos

Nativa, Sinop, v. 8, n. 4, p. 538-543, jul./ago. 2020. 
microrganismos ruminais ao conteúdo celular. De acordo com France et al. (1990) a produção de gases fornece informações relativas à disponibilidade do substrato.

Isso explica a queda na DIVMS da dieta com inclusão de FGMD a partir de $200 \mathrm{~g} \mathrm{~kg}^{-1}$, pois conforme a adição do coproduto, houve aumento na fração fibrosa e redução dos carboidratos não fibrosos na dieta. O que também explica a redução do VGtotal, pois com redução na digestibilidade, consequentemente terá uma menor produção de gases.

Já os níveis de inclusão do DDGM não influenciaram a DIVMS e consequentemente a VGtotal, em função da não redução da KdCHOF e produção de gás dessa fração, em que se mantiveram em condições que não reduzissem a DIVMS e a produção total de gases.

A mesma explicação pode ser dada para os efeitos das inclusões do FG, porém em função dos níveis de $\mathrm{KdCNF}$ e a VGCNF, ou seja, da fração não fibrosa da dieta.

\section{CONCLUSÕES}

As inclusões dos diferentes coprodutos alteraram alguns dos parâmetros de degradação avaliados, porém, somente para o gérmen de milho desengordurado é que houve redução na digestibilidade in vitro da MS e produção de gás total. Desta forma, para o grão seco da destilaria do milho e o farelo de girassol pode se incluir até o nível de $300 \mathrm{~g} \mathrm{~kg}^{-1}$ nas dietas, e para o farelo de gérmen de milho desengordurado até $100 \mathrm{~g} \mathrm{~kg}^{-1}$.

\section{REFERÊNCIAS}

ARMENTANO, L.; PEREIRA, M. Measuring the effectives of fiber by animal trial. Journal of Dairy Science, Champaign, v. 80 , n. 7 , p. 1416-1425, 1997. DOI: https://dx.doi.org/10.3168/jds.S0022-0302(97)76071-5

AOAC_ASSOCIATION OF OFFICIAL ANALYTICAL CHEMISTS. Official methods of analysis. 15. ed. Washington: AOAC. 1990. 771 p.

DETMANN, E.; SOUZA, M. A.; VALADARES FILHO, S. C.; QUEIROZ, A. C.; BERCHIELLI, T. T.; SALIBA, E. O. S, CABRAL, L. S.; PINA, D. S.; LADEIRA, M. M.; AZEVEDO, J. A. G. Métodos para análise de alimentos. 2. ed. Visconde do Rio Branco: Suprema, 2012. $214 \mathrm{p}$.

FRANCE, J.; THORNLEY, J. H. M.; LOPEZ, S.; SIDDONS, R. C.; DHANOA, M. S.; VAN SOEST, P. J.; GILL, M. On the two-compartment model for estimating extent of feed degradation in the rumen. Journal of Theoretical Biology, London, v. 146, n. 2, p. 269-287, 1990. DOI: https://dx.doi.org/10.1016/S00225193(05)80139-0

GOES, R. H. T. B.; SOUZA, K. A.; PATUSSI, R. A.; CORNELIO, T. C.; OLIVEIRA, E. R.; BRABES, K. C. S. Degradabilidade in situ dos grãos de crambe, girassol e soja, e de seus coprodutos em ovinos. Acta Scientiarum, Maringá, v. 32, n. 3, p. 271-277, 2010. DOI: http://dx.doi.org/10.4025/actascianimsci.v32i3.7913

HALL, M. B. Challenges with non-fiber carbohydrate methods. Journal of Animal Science, Champaign, v. 81, n. 12 , p. $3226-3232$, 2003. DOI: https://dx.doi.org/10.2527/2003.81123226x

HUHTANEN, P.; SEPPÄLÄ, A.; OTS, M.; AHVENJÄRVI, S.; RINNE, M. In vitro gas production profiles to estimate extent and effective first-order rate of neutral detergent fibre digestion in the rumen. Journal of Animal Science, v. 86, n. 3 , p. 651-659, 2008. DOI: https://dx.doi.org/10.2527/jas.2007-0246

LICITRA, G.; HERNANDEZ, T. M.; VAN SOEST, P. J. Standardization of procedures for nitrogen fractionation of ruminant feeds. Animal Feed Science Technology, v. 57, n. 4 , p. 347-358, 1996, DOI: https://dx.doi.org/10.1016/0377-8401(95)00837-3

LOUSADA JUNIOR, J. E.; NEIVA, J. N. M.; RODRIGUEZ, N. M.; PIMENTEL, J. C. M.; LÔBO, R. N. B. Consumo e digestibilidade de subprodutos do processamento de frutas em ovinos. Revista Brasileira de Zootecnia, Viçosa, v. 34, n. 2, p. 659-669, 2005.

MCDOUGALL, E. I. Studies on ruminant saliva. 1. The composition and output of sheep's saliva. Biochemical Journal, v. 43, n. 1, p. 99-109, 1949. DOI: https://dx.doi.org/10.1042/bj0430099

MALAFAIA, P. Taxas de digestão das frações protéicas e de carboidratos de alimentos por técnicas "in situ", "in vitro" e de produção de gases. Viçosa MG: UFV, 1997. 85f. Tese (Doutorado em Zootecnia) Universidade Federal de Viçosa, Viçosa, 1997.

NRC. Nutrient requirements of small ruminants. Washington (DC): National Academia of Science, 2007. 384 p.

PAIXÃO, M. L. Desempenho produtivo e exigências nutricionais de bovinos de corte em pastagens de Brachiaria decumbens com suplementação proteica. 2008. 124 p. Tese (Doutorado em Zootecnia) - Programa de Pós Graduação em Zootecnia, Universidade Federal de Viçosa, Viçosa, 2008.

PELL, A. N.; SCHOFIELD, P. Computerized Monitoring of Gas Production to Measure Forage Digestion in Vitro. Journal Dairy Science, v. 76, p. 1063-1073. 1993. DOI: https://dx.doi.org/10.3168/jds.S0022-0302(93)77435-4

SANTO, A. X.; SILVA, L. D. F.; LANÇANOVA, J. A. C.; RIBEIRO, E. L. A.; MIZUBUTI, I. Y.; FORTALEZA, A. P. S.; HENZ, É. L.; JUNIOR, F. L. M. Fracionamento de carboidratos e proteínas, cinética de degradação ruminal in vitro pela técnica de produção de gás, de rações suplementares contendo torta de girassol, Arquivo Brasileiro de Medicina Veterinaria e Zootecnia, Belo Horizonte, v. 69, n. 1, p. 234-242, 2017. DOI: http://dx.doi.org/10.1590/1678-4162-8761

SCHOFIELD, P.; PITT, R. peE.; PELL, A. N. Kinetics of fiber digestion from in vitro gas productio". Journal of Animal Science, Champaign, v. 72, n. 11, p. 2980-2991. 1994 DOI: https://dx.doi.org/10.2527/1994.72112980x

THEODOROU, M. K.; WILLIAMS, B. A.; DHANOA, M. S.; MCALLAN, A. B.; FRANCE, J. A simple gas production method using a pressure transducer to determine the fermentation kinetics of ruminant feed. Animal Feed Science Technology, v. 48, n.1, p. 185197, 1994

TILLEY, J. M. A.; TERRY, R. A. A two-stage technique for the in vitro digestion of forage Crops. The Journal of the British Grassland Society, v. 18, n. 2, p. 104-111, 1963. DOI: $\quad$ https://dx.doi.org/10.1111/J.13652494.1963.tb00335.x 